\title{
Significant Differences in Media Components and Predicted Growth Rates of 58 Escherichia coli Genome-scale Models
}

\section{Felicia Leyi Tan, Zhi Jue Kuan, Nabil Amir-Hamzah, Xander Kng, Yik Yew Wee, Si Xian Sor and Maurice HT Ling*}

School of Applied Sciences, Temasek Polytechnic, Singapore

*Corresponding Author: Maurice HT Ling, School of Applied Sciences, Temasek Polytechnic, Singapore.
Received: January 03, 2022

Published: January 27, 2022

(c) All rights are reserved by Maurice HT

Ling., et al.

\begin{abstract}
Escherichia coli is a common host for metabolite production and genome-scale metabolic models (GSMs) is an important computational tool to aid in such experimental design. As of September 30, 2021; 58 GSMs have been registered with BiGG database. However, these GSMs had been built for different applications and no large-scale comparative study had been performed to-date. In this study, we examine the media components and predicted growth rates of these $58 \mathrm{GSMs}$ using flux balance analysis across various glucose uptake rates. Only 5 out of 29 uptake rates (as proxy for media components) are common in all 58 GSMs; namely, proton, water, ammonium, oxygen, and phosphate. $74.25 \%$ (2370 of the 3192) pairwise comparisons of predicted growth rates show significant differences ( $p$-value $<0.05$ ) and 34 of 42 pairwise comparisons of predicted growth rates within the same strain are significantly different. Hence, our results demonstrated substantial differences in media components and significant differences in predicted growth rates between the GSMs and even within GSMs constructed for the same strain.
\end{abstract}

Keywords: Escherichia coli; Genome-scale Metabolic Models (GSMs); Ammonium

\section{Introduction}

Escherichia coli is a fast-growing bacterium in chemically defined media and with extensive molecular tools available [1], it has been the linchpin in discovering many important findings in molecular biology and cell physiology [2]. The emergence of $E$. coli as a notable host for natural product biosynthesis [2] also led to successful engineering of $E$. coli for metabolite production [3]. However, a challenge faced by metabolic engineering is the complexity of pathway optimization $[4,5]$. This is due to the myriad of regulatory systems that control natural metabolic pathways. The precise control required over the expression of several natural and heterologous genes to avoid limiting the desired product yield [6] is an arduous process when done manually. Furthermore, each optimization is dependent on multiple factors such as the pathway and compound, making this a multivariate problem that poses as a difficulty to many researchers $[7,8]$.

A solution to this challenge involves the usage of a GenomeScale Metabolic Model (GSM), which is a fundamental framework built upon extensive collection and curation of biological data of gene annotation, gene functions, metabolites, metabolic reactions, enzymes, and their interactions inside a targeted organism [9-12]. These GSMs can be analyzed using computation algorithms such as constraint-based flux balance analysis (FBA) to comprehend the functions and objectives of the metabolic network [13]. Therefore, GSMs can be an in silico platform for lessening the difficulty and burden on the researchers [7], and had been used in many studies [12]. For example, iBsu1144 (a Bacillus subtilis GSM) was used to identify the effects of oxygen transfer rates on the production of serine alkaline protease and recombinant proteins [14], and iEK1101 (a Mycobacterium tuberculosis GSM) was used to study the metabolic status of M. tuberculosis under hypoxic conditions [15].

Since the $1^{\text {st }}$ E. coli GSM, iJE660 [16], many E. coli GSMs have been developed with increasing availability of experimental data. The most recent $E$. coli GSM is the iML1515 model, which has been used to maximize lysine production [17]. Another commonly used model is iAF160 which had been used in various studies [18-20] including to optimize the yield of violacein [21]. As of September 30, 2021; BiGG database [22] lists 58 E. coli GSMs ${ }^{1}$. Hence, it is difficult to know which model to use, and what the similarities and differences are in terms of media components and predicted growth rate. Here, we review, and differentiate these 58 E. coli GSMs. Our results demonstrated substantial differences in media components and significant differences in predicted growth rates between the GSMs and even within GSMs constructed for the same strain.

${ }^{1}$ http://bigg.ucsd.edu/search?query=Escherichia+coli 
Materials and Methods

Models

58 GSMs from BiGG database [22]; namely, (A) iJR904, (B) iAF1260, (C) e_coli_core, (D) iAF1260b, (E) iJ01366, (F) iEC042_1314, (G) iECP_1309, (H) iEC55989_1330, (I) iECABU_ c1320, (J) iAPEC01_1312, (K) iEcolC_1368, (L) iECB_1328, (M) iB21_1397, (N) iECD_1391, (O) iECBD_1354, (P) iBWG_1329, (Q) ic_1306, (R) iECDH1ME8569_1439, (S) iEcDH1_1363, (T) iECED1_1282, (U) iETEC_1333, (V) iEcHS_1320, (W) iECIAI1_1343, (X) iECIAI39_1322, (Y) iECOK1_1307, (Z) iEK011_1354, (AA) iLF82_1304, (AB) iECNA114_1301, (AC) iEC0103_1326, (AD) iEC0111_1330, (AE) iE2348C_1286, (AF) iEcE24377_1341, (AG) iECH74115_1262, (AH) iZ_1308, (AI) iECs_1301, (AJ) iECSP_1301, (AK) iECO26_1355, (AL) iG2583_1286, (AM) iNRG857_1313, (AN) iECS88_1305, (AO) iECSE_1348, (AP) iECSF_1327, (AQ) iEcSMS35_1347, (AR) iECDH10B_1368, (AS) iY75_1357, (AT) iEC1372_W3110, (AU) iUMN146_1321, (AV) iECUMN_1333, (AW) iUMNK88_1353, (AX) iUTI89_1310, (AY) iWFL_1372, (AZ) iECW_1372, (BA) iEC1349_Crooks, (BB) iEC1356_Bl21DE3, (BC) iEC1344_C, (BD) iEC1368_DH5a, (BE) iEC1364_W, and (BF) iML1515; were used.

\section{Comparison of media components}

Media components for each GSM could be extracted using Cameo [7], which was available via cameo-medium-cpds command from AdvanceSyn Toolkit [8], and compared across GSMs for commonality.
Comparison of growth rates

Predicted growth rates at various glucose intakes $(10,9,8,7,6$, $5,4,3,2$, and 1 millimole per gram dry weight per hour of glucose intake) were given as proxy as output from the objective function [23] after flux balance analysis [24] using Cameo [7], which was available via cameo-medium-fba command from AdvanceSyn Toolkit [8], by changing the glucose uptake rates (EX_glc_D_e) while maintaining the rest of the media components unchanged. Predicted growth rates from each GSM were normalized into normalized predicted growth rates by calculating each predicted growth rate as a ratio of predicted growth rate at 10 millimole per gram dry weight per hour of glucose intake. Normalized predicted growth rates between any two GSMs were compared using paired t-test to identify differences in predicted growth rates across different glucose uptake rates under the null hypothesis of no average differences in predicted growth rates.

\section{Results and Discussion}

A cursory examination suggests that these 58 GSMs were built for specific purposes (Table 1), such as metabolite production [25] or examining gene essentiality [26], or for specific strains, such as E. coli APEC 01 [27] or E. coli W3110 [28]. However, a review of the applications of each GSM is likely to be extensive not the intent of this study as we are interested only in similarities and differences of each GSM in terms of media components, predicted growth rate and fluxes.

\begin{tabular}{|c|c|}
\hline GSM Model IDs & Purposes and Applications \\
\hline (A) iJR904 & $\begin{array}{l}\text { Integrate and analyze the diverse datasets, such as 'omics' data and to provide a more chemically accurate } \\
\text { description of E. coli metabolism over GSM iJE660a [29]. } \\
\text { Investigating the predictive power of the constraint-based modelling approach of flux distribution in com- } \\
\text { parison to the kinetic modelling approach [30]. } \\
\text { Test DEF, an automated gap filling approach based on the endosymbiosis theory to fill gaps and gain more } \\
\text { insights for the genomic annotation and modelling [31]. }\end{array}$ \\
\hline (B) iAF1260 & $\begin{array}{l}\text { Based off iJR904, iAF1260 serves as a BIGG database as it contains the current knowledge of E. coli metabo- } \\
\text { lism and this GSM is also a framework for mathematical analysis and computational predictions. iAF1260 } \\
\text { showed an overall increase of } 4 \text { and 16\% over iJR904 predictions provide a broader perspective of cellular } \\
\text { metabolism for E. coli [32]. } \\
\text { Investigate the efficiency of GridProd [33], a method of calculating parsimonious metabolic networks and the } \\
\text { production of metabolites while involving several reactions that were included in the iAF1260 model. } \\
\text { Repurposed into an extended version of the model, iAF1260vio, to include violacein production [21]. }\end{array}$ \\
\hline (C) e_coli_core & $\begin{array}{l}\text { Subset of the iAF1260 containing the central metabolism of E. coli [34]. } \\
\text { Repurposed as a stoichiometric model by adding eight different pathways to produce butanol [35]. } \\
\text { Used in comparison to the iAF1260 model to test a new pessimistic optimization framework for the identi- } \\
\text { fication of the optimal knock out strategies for maximum targeted bio-production under model uncertainty } \\
\text { and have derived the robustness and stability of the metabolic strain perturbation through the modelling } \\
\text { [36]. }\end{array}$ \\
\hline
\end{tabular}


\begin{tabular}{|l|l|}
\hline (D) iAF1260b & A slightly improved and updated version of the iAF1260 to address incorrect model predictions and most
\end{tabular} genes in the iAF1260b model were experimentally determined for conditional essentiality [26,37].

(E) ij01366

Updated version of iAF1260 to include newly characterized genes and reactions where gaps in iAF1260 network were identified [26].

Used to evaluate RIPTiDe (Reaction Inclusion by Parsimony and Transcript Distribution), a method that utilizes the transcriptomic abundances and parsimony of overall flux for the identification of the most costeffective usage of metabolism reflecting the cell's investment into transcription with further addition of identifying the activity of context-specific metabolic pathways without knowledge of the media conditions [38].

Comparison with its repurposed versions created from different methods and tool developments to identify key properties and differences; thus, emphasizing the importance of considering enzyme constraints in enhancements [39].

(F) iEC042_1314 GSM for E. coli 042 [27].

\begin{tabular}{|c|c|}
\hline (G) iECP_1309 & GSM for E. coli 536 [27]. \\
\hline
\end{tabular}

\begin{tabular}{l|c} 
(H) iEC55989_1330 & GSM for E. coli 55989 [27]. \\
\hline
\end{tabular}

(I) iECABU_c1320

(J) iAPEC01_1312 GSM for E. coli ABU 83972 [27] and used to analyze probiotic E. coli Nissle 1917 [40].

(K) iEcolC_1368 GSM for E. coli APEC 01 [27].

\begin{tabular}{|l}
\hline (L) iECB_1328 \\
\hline (M) iB21_1397 \\
\hline (N) iECD_1391 \\
\hline (O) iECBD_1354 \\
\hline (P) iBWG_13
\end{tabular}
production and growth rates [25].

GSM for E. coli B str. REL606 [27] and used to examine adaptive evolution of carbon source utilization [41].

(P) Model growth rates of the E. coli strain BL21(DE3) [42]. GSM for E. coli BL21(DE3) [43]. GSM for E. coli 'BL21-Gold(DE3)pLysS AG' strain [27].

(Q) ic_1306 GSM for E. coli BW2952 [44]. GSM for uropathogenic E. coli CFT073 [27].

iECDH1ME8569_1439

(S) iEcDH1_1363

(T) iECED1_1282

(U) iETEC_1333 GSM for E. coli DH1 [27].

(V) iEcHS_1320

(W) iECIAI1_1343

(X) iECIAI39_1322

(Y) iECOK1_1307

(Z) iEK011_1354

(AA) iLF82_1304

(AB) iECNA114_1301

(AC) iEC0103_1326

(AD) iEC0111_1330

(AE) iE2348C_1286

(AF) iEcE24377_1341

(AG) iECH74115_1262

(AH) iZ_1308

GSM for E. coli 0157:H7 str. EDL933 [27] and has been utilised in gene knock out optimization and is compa-

rable quantitatively with the model iJ01366 [46].

(AI) iECs_1301

GSM for enterohemorrhagic E. coli 0157:H7 str. Sakai and has been used to test the robustness of the GSMs [47].

(AJ) iECSP_1301 GSM for E. coli DH1 [27] for fatty acids production [45] GSM for E. coli ED1a [27].

GSM for E. coli ETEC H10407 [27]. GSM for E. coli HS [27]. GSM for E. coli IAI1 [27]. GSM for E. coli IAI39 [27]. GSM for E. coli IHE3034 [27]. GSM for E. coli K011FL [27]. GSM for E. coli LF82 [27]. GSM for E. coli NA114 [27]. GSM for E. coli 026:H11 str. 11368 [27]. GSM for E. coli 0111:H- str. 11128 [27]. GSM for E. coli 0127:H6 str. E2348/69 [27]. GSM for E. coli 0139:H28 str. E24377A [27].

GSM for E. coli 0157:H7 str. EC4115 [27].
GSM for enterohemorrhagic E. coli 0157:H7 str. TW14359 [27]. 


\begin{tabular}{|c|c|}
\hline$(\mathrm{AK})$ iECO26_1355 & GSM for E. coli 026:H11 str. 11368 [27]. \\
\hline (AL) iG2583_1286 & GSM for E. coli 055:H7 str. CB9615 [27]. \\
\hline (AM) iNRG857_1313 & GSM for E. coli 083:H1 str. NRG 857C [27]. \\
\hline (AN) iECS88_1305 & GSM for E. coli S88 [27]. \\
\hline$(\mathrm{AO})$ iECSE_1348 & GSM for E. coli SE11 [27]. \\
\hline (AP) iECSF_1327 & GSM for E. coli SE15 [27]. \\
\hline (AQ) iEcSMS35_1347 & GSM for E. coli S-M-S-3-5 [27]. \\
\hline (AR) iECDH10B_1368 & GSM for E. coli K-12 str. DH10B [27]. \\
\hline (AS) iY75_1357 & GSM for E. coli K-12 str. W3110 for production of 2-keto-4-hydroxybutyrate and 1,3-propanediol [28]. \\
\hline (AT) iEC1372_W3110 & GSM for E. coli K-12 str. W3110 [27]. \\
\hline (AU) iUMN146_1321 & GSM for E. coli UM146 [27]. \\
\hline$(\mathrm{AV})$ iECUMN_1333 & GSM for E. coli UMN026 [27]. \\
\hline (AW) iUMNK88_1353 & GSM for E. coli UMNK88 [27]. \\
\hline (AX) iUTI89_1310 & GSM for E. coli UTI89 [27]. \\
\hline (AY) iWFL_1372 & GSM for E. coli W [27]. \\
\hline (AZ) iECW_1372 & GSM for E. coli W [27]. \\
\hline (BA) iEC1349_Crooks & GSM for E. coli ATCC 8739 [48]. \\
\hline (BB) iEC1356_Bl21DE3 & GSM for E. coli BL21 (DE3) [48]. \\
\hline (BC) iEC1344_C & GSM for E. coli C [48]. \\
\hline (BD) iEC1368_DH5a & GSM for E. coli DH5a [48]. \\
\hline (BE) iEC1364_W & GSM for E. coli $\mathrm{W}[48]$. \\
\hline (BF) iML1515 & $\begin{array}{l}\text { Most complete genome-scale reconstruction of the metabolic network in E. coli K-12 MG1655 to carry } \\
\text { out a comparative structural proteome analysis of } 1122 \text { E. coli strains and identify multi-strain sequence } \\
\text { variations, as well as providing a knowledge base for integrating modelling framework bridging systems and } \\
\text { structural biology linked to } 1515 \text { protein structures. } \\
\text { Investigate the creation of extended metabolic models through augmenting the iML1515 model with reac- } \\
\text { tions from promiscuous enzyme activity using PROXIMAL and EMMA as a prediction tool and a way to com- } \\
\text { pare the generated putative derivatives with a set of metabolites documented in ECMDB [50]. } \\
\text { Repurposed as an enzyme-constrained model, ec_iML1515, using GECKO method to assist in the clarifica- } \\
\text { tion of intracellular mechanisms and improve the production titre of lysine while accurately predicting and } \\
\text { designing cellular phenotypes [17]. }\end{array}$ \\
\hline
\end{tabular}

Table 1: Purposes and Applications of Each GSM.

\section{Number of metabolites and reactions}

The average number of metabolites (Figure 1A, and 1B; $5 \%$ trimmed mean) is 1898. Models iWFL_1372 [27] and iECW_1372 [27] have the highest number of metabolites, 1973 metabolites; while e_coli_core has the lowest number of metabolites, 72 metabolites. The average number of reactions (Figure 1C, and 1D; 5\% trimmed mean) is 2697 , with the highest $(\mathrm{n}=2782)$ in iWFL_1372 [27] and iECW_1372 [27], and lowest $(n=95)$ in e_coli_core [34]. Model e_coli_core is the subset of iAF1260 [32], containing only the central metabolism of E. coli [34], which is often used for educational purposes [51] and tool testing [52-54]. Another notable left skewed number would be 761 metabolites found in iJR904 [29], which was used to integrate and analyse the diverse datasets and to provide a more chemically accurate description of $E$. coli metabolism over GSM iJE660a. The average number of genes (Figure $1 \mathrm{E}$, and $1 \mathrm{~F} ; 5 \%$ trimmed mean) is 1322, with iML1515 [49] being the highest $(\mathrm{n}=1516)$ and e_coli_core [34] being the lowest $(\mathrm{n}=$ 137). Model iML1515 [49] is the most recent E. coli GSM model catalogued in BiGG [22], and there is a trend of increasing number of genes over the years - from 904 genes in iJR904 [29] to 1515 genes in iML1515 [49]. Hence, it is likely that this trend will continue as iML1515 [49] contains only $34.4 \%$ of the 4401 genes in $E$. coli $\mathrm{K}-12$ genome [55]. 

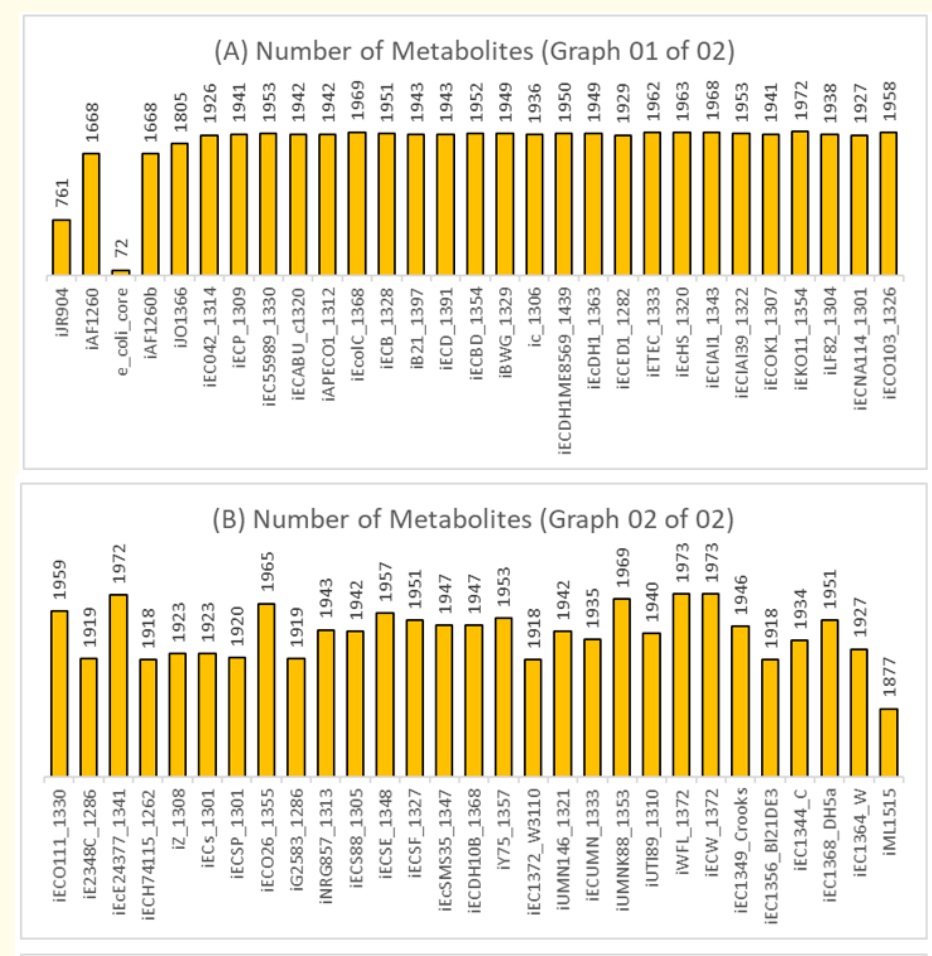

(C) Number of Reactions (Graph 01 of 02)
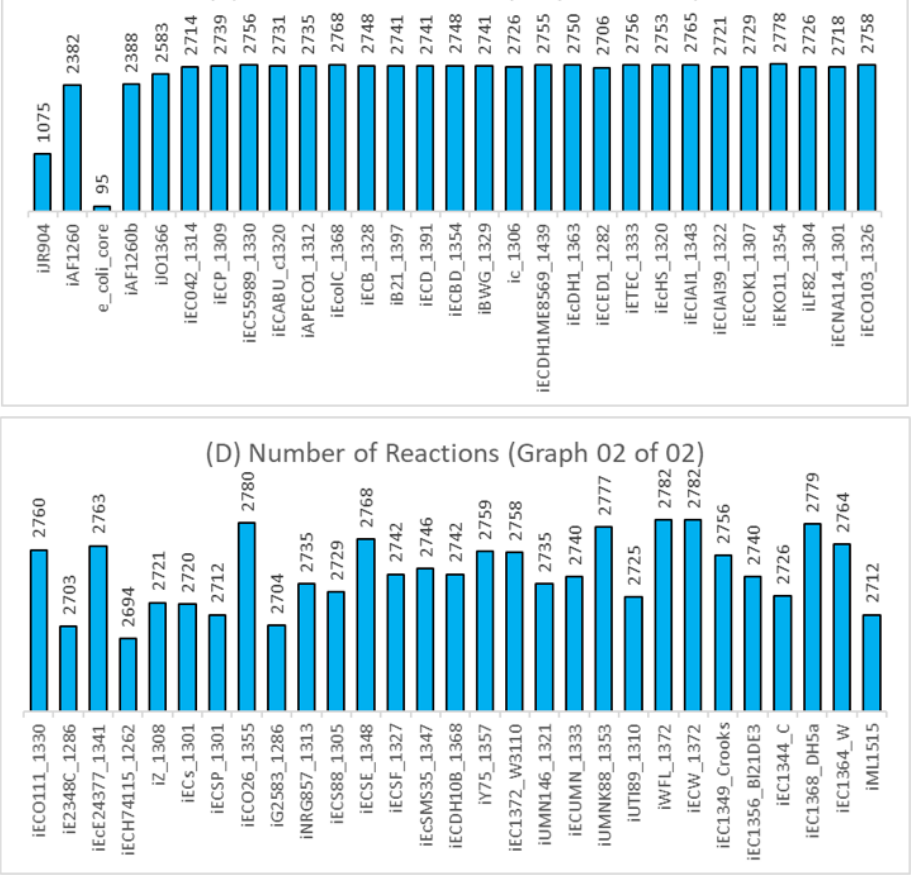

(E) Number of Genes (Graph 01 of 02)

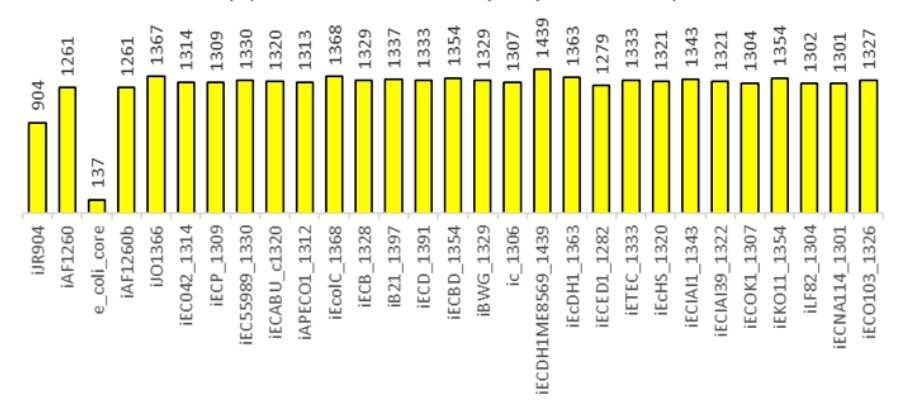




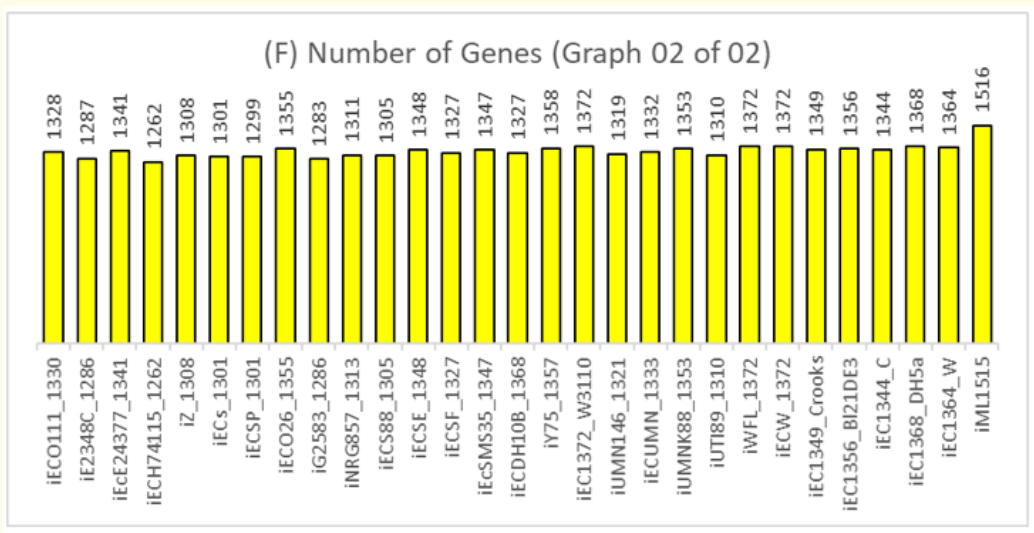

Figure 1: Statistics (Number of Metabolites, Reactions, and Genes) in Each GSM. The GSMs are arranged by year, from earliest (iJR904) to latest (iML1515).

\section{Five media components are common in All GSMs}

Five media components are common in all GSMs (Table 2) out of 29; namely, EX_h_e (proton), EX_h20_e (water), EX_nh4_e (ammonium), EX_o2_e (oxygen), and EX_pi_e (phosphate); all of which can be found in M9 minimum media [18] under oxic environments. This suggests that these 5 components are critical for aerobic functions of E. coli. Not taking account of core metabolism (e_coli_core) and the earliest GSM (iJR904), 14 other media components are common in the remaining 56 GSMs.

Of which, 6 media components (calcium, chloride, magnesium, potassium, sodium, sulphate] are found in M9 minimum media. The remaining 8 media components (copper, cobalt, ferrous ion, ferric ion, manganese, molybdate, tungstate, and zinc) were known to affect $E$. coli growth. Copper alone shows slight growth inhibition of E. coli 0157:H7 but more pronounced growth inhibition in the presence of lactic acid [56]. Cobalt has been shown to induce stress to E. coli [57] and toxicity at high concentration [58]. An early study by Ratledger and Winder 1964 had demonstrated effects or iron (ferric and ferrous ions) and zinc on E. coli growth [59] and the growth supporting effects of iron had also been re-demonstrated in a more recent study [60]. The presence of manganese is suggested to have a protective effect of $E$. coli to high oxidative stress [61] by activation of manganese-superoxide dismutase [62]. Similarly, molybdate [63] and tungstate [64] has also been shown to inhibit $E$. coli growth.

Substantial differences in the media components in various models lends difficulties in conducting complete comparative study such as that of Cheong., et al. [18] across multiple GSM mod- els. As a result, only the effects of glucose on growth across models is studied. However, it is surprising that glucose (EX_glc_D_e) is not found in iECIAI1_1343, which is GSM for E. coli IAI1 [27]. As we are interested in examining the predicted growth rate and fluxed under varying glucose condition, iECIAI1_1343 was removed from study as the model cannot respond to glucose.

On the other hand, Ex_leu_L_e (leucine), EX_thm_e (thiamine), and Ex_trp_L_e (tryptophan) is found in only one GSM each: iECDH10B_1368 [27], iECIAI39_1322 [27], and ic_1306 [27] respectively. The reason for having these specific nutrients in the media is due to auxotrophic nature of these strains from experimental or predicted findings. Leucine is found only in iECDH10B_1368, a GSM for E. coli K-12 substr. DH10B [27], which lacks leucine synthesis pathway; thus, requires leucine for growth on minimal medium [65]. Thiamine is found only in iECIAI39_1322, a GSM for E. coli IAI39, which is predicted to lack thiamine synthesis [27]. This has resulted in the routine addition of thiamine into minimum media to support growth [66]. Tryptophan is found only in ic_1306, a GSM for uropathogenic E. coli CFT073, which is similarly to be predicted to be auxotrophic to tryptophan [27]. This is partly supported by an earlier study suggesting that E. coli CFT073 catalyzes tryptophan during serine and aspartate depletion [67].

Substantial differences between predicted growth rates from varying glucose uptake rates across 58 GSMs

Of the 3192 pairwise permutation of possible non-self-comparison paired t-tests (Figure 2), 74.25\% ( $\mathrm{n}=2370)$ are significant (p-value < 0.05); of which, 2036 paired t-tests are highly significant ( $\mathrm{p}$-value $<0.01)$. Hence, only $25.75 \%(\mathrm{n}=822)$ are not sig- 
nificant ( $\mathrm{p}$-value > 0.05). This suggests substantial differences between normalized predicted growth rates across the 57 GSMs. As a whole, the average number of GSMs not significantly different from a specific GSM in terms of predicted growth rate is 14.4 (Figure 3), with iECP_1309 [27] and ic_1306 [27] having the least differences (not significantly different with 49 other GSMs) and most differences (significantly different from all 57 other GSMs) respectively. In terms of phylogram analysis based on Manhattan distance [68], it is interesting to note that iAF1260 [32] and iAF1260b [26,37] are most different to the other 56 GSMs (Figure 4). However, iAF1260b [26,37] can be seen as a slight improvement and correction of iAF1260 [32]; hence, not surprising that they are clustered together. Nevertheless, these results suggest significant differences between the 58 examined GSMs. This suggests that GSMs of the same species may vary substantially, which is supported Nouri., et al. [69] compared the prediction of 3 GSMs for Zymomonas mobilis ZM4 and reported demonstrating differences between various models of the same species.

\begin{tabular}{|c|c|c|}
\hline Media Components & Name & Found or Not Found in GSMs in this Review \\
\hline EX_h_e & $\mathrm{H}^{+}$exchange & Found in all GSMs \\
\hline EX_h2o_e & $\mathrm{H}_{2} \mathrm{O}$ exchange & Found in all GSMs \\
\hline EX_nh4_e & Ammonia exchange & Found in all GSMs \\
\hline EX_o2_e & $\mathrm{O}_{2}$ exchange & Found in all GSMs \\
\hline EX_pi_e & Phosphate exchange & Found in all GSMs \\
\hline EX_co2_e & $\mathrm{CO}_{2}$ exchange & Found in all GSMs except iECIAI1_1343 \\
\hline EX_fe2_e & $\mathrm{Fe}^{2+}$ exchange & Found in all GSMs except e_coli_core \\
\hline EX_glc_D_e & D-Glucose exchange & Found in all GSMs except iECIAI1_1343 \\
\hline EX_k_e & $\mathrm{K}^{+}$exchange & Found in all GSMs except e_coli_core \\
\hline EX_na1_e & Sodium exchange & Found in all GSMs except e_coli_core \\
\hline EX_so4_e & Sulfate exchange & Found in all GSMs except e_coli_core \\
\hline EX_ca2_e & Calcium exchange & Found in all GSMs except e_coli_core, and iJR904 \\
\hline EX_cl_e & Chloride exchange & Found in all GSMs except e_coli_core, and iJR904 \\
\hline EX_cobalt2_e & $\mathrm{Co}^{2+}$ exchange & Found in all GSMs except e_coli_core, and iJR904 \\
\hline EX_cu2_e & $\mathrm{Cu}^{2+}$ exchange & Found in all GSMs except e_coli_core, and iJR904 \\
\hline EX_fe3_e & $\mathrm{Fe}^{3+}$ exchange & Found in all GSMs except e_coli_core, and iJR904 \\
\hline EX_mg2_e & Mg exchange & Found in all GSMs except e_coli_core, and iJR904 \\
\hline EX_mn2_e & $\mathrm{Mn}^{2+}$ exchange & Found in all GSMs except e_coli_core, and iJR904 \\
\hline EX_mobd_e & Molybdate exchange & Found in all GSMs except e_coli_core, and iJR904 \\
\hline EX_tungs_e & Tungstate exchange & Found in all GSMs except e_coli_core, and iJR904 \\
\hline EX_zn2_e & Zinc exchange & Found in all GSMs except e_coli_core, and iJR904 \\
\hline EX_cbl1_e & Cob(I)alamin exchange & Found in all GSMs except e_coli_core, iJR904, iML1515, and iECIAI1_1343 \\
\hline EX_ni2_e & $\mathrm{Ni}^{2+}$ exchange & Found in all GSMs except e_coli_core, iJR904, iAF1260, and iAF1260b \\
\hline EX_sel_e & Selenate exchange & Found in all GSMs except e_coli_core, iJR904, iAF1260, and iAF1260b \\
\hline EX_slnt_e & Selenite exchange & Found in all GSMs except e_coli_core, iJR904, iAF1260, and iAF1260b \\
\hline EX_nac_e & Nicotinate exchange & Not found in all GSMs except iECDH10B_1368, and iECUMN_1333 \\
\hline EX_leu__L_e & L-Leucine exchange & Not found in all GSMs except iECDH10B_1368 \\
\hline EX_thm_e & Thiamin exchange & Not found in all GSMs except iECIAI39_1322 \\
\hline EX_trp__L_e & L-Tryptophan exchange & Not found in all GSMs except ic_1306 \\
\hline
\end{tabular}

Table 2: Comparison of Media Components. 


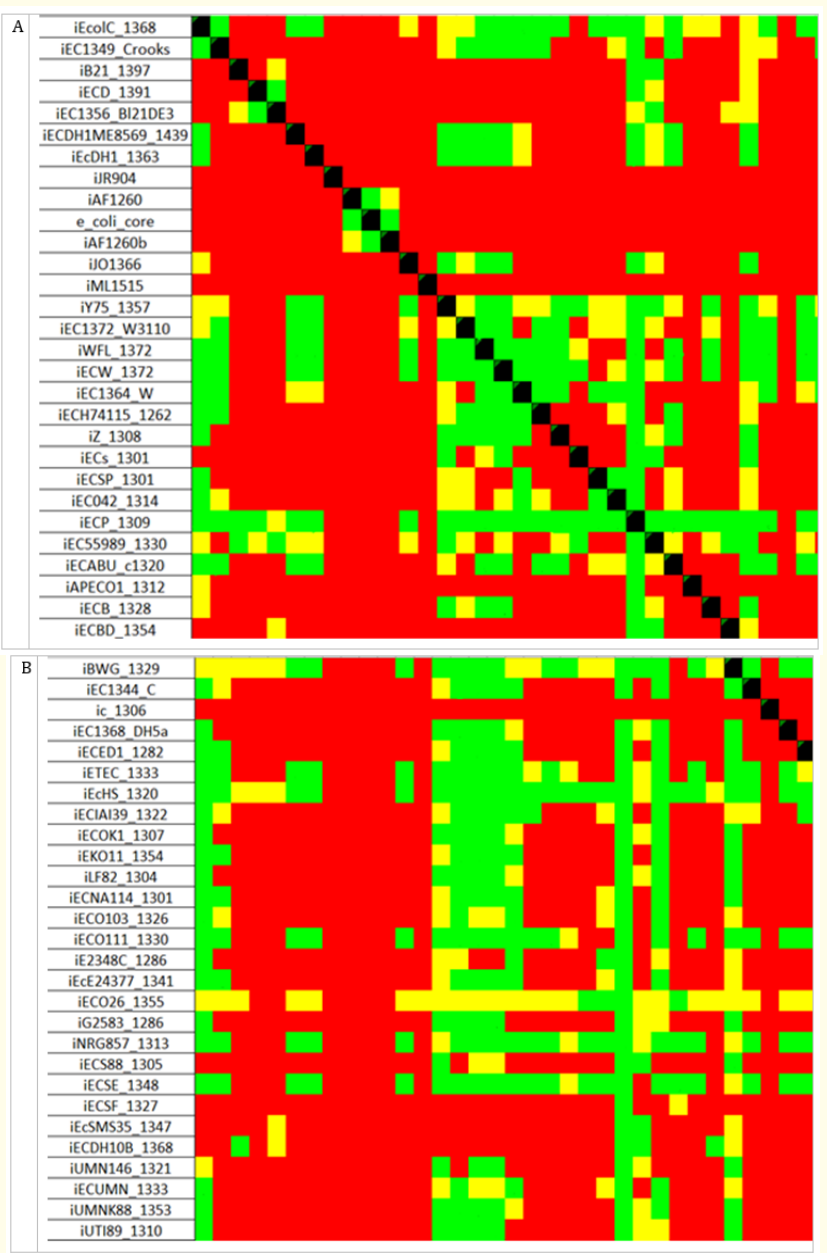

Figure 2: 3192 Paired t-test P-values of Predicted Normalized Growth Rates Across 57 GSMs. Panel A and B are consecutive images. Green represents p-value more than 0.05. Yellow represents p-value between 0.01 and 0.05 . Red represents p-value less than 0.01 . Black represents self-comparison.

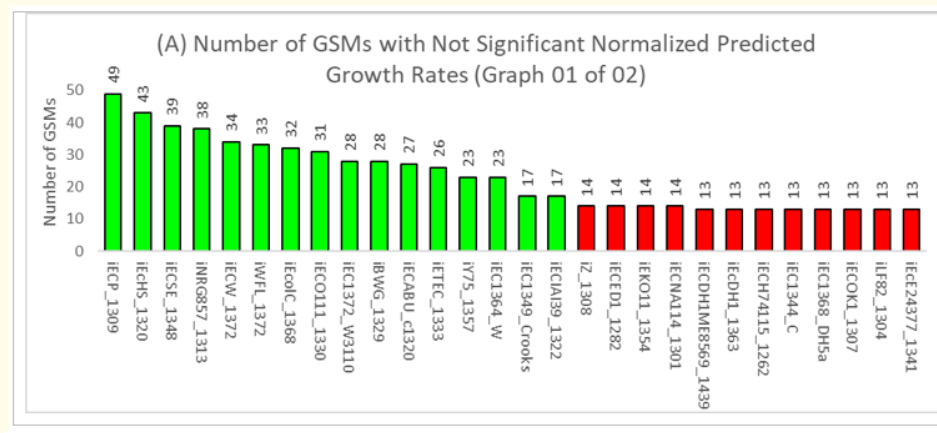

(B) Number of GSMs with Not Significant Normalized Predicted

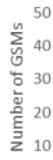
Growth Rates (Graph 02 of 02)

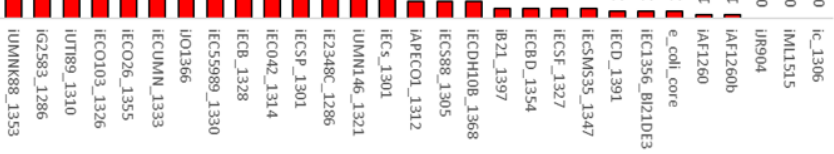

Figure 3: Number of GSMs Not Significantly Different to a Specific GSM by Normalized Predicted Growth Rates. The average number of non-significances is 14.4; hence, the green bars are GSMs with more similarity than average while the red bars are GSMs with less similarity than average in terms of normalized predicted growth rates. 


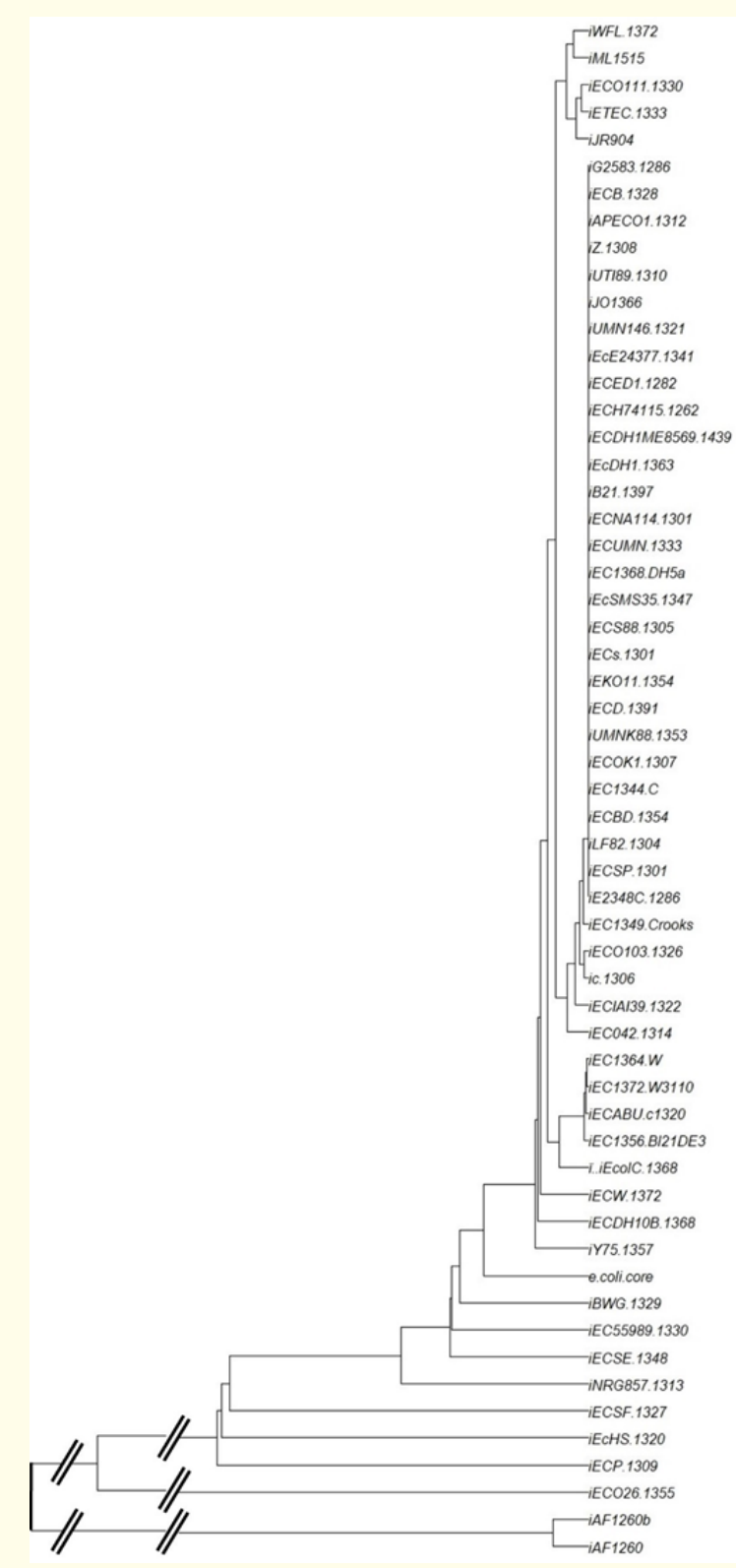

Figure 4: Phylogram of GSMs Based on Normalized Growth Rates. Distance matrix was calculated using Manhattan distance [68].

34 of 42 pairwise comparisons show substantial differences between predicted growth rates from GSMs for the same strain

Paired t-test analyses between predicted growth rates within the same strain demonstrated 34 of the 42 paired t-tests to be significant (Table 3). The two GSMs (iEcolC_1368, and iEC1349_Crooks) for $E$. coli ATCC 8739 shows insignificant difference in normalized predicted growth rate (p-value $=0.350$ ). Of the 3 GSMs (iB21_1397, iECD_1391, and iEC1356_Bl21DE3) for E. coli BL21(DE3), only between iECD_1391 and iEC1356_Bl21DE3 is not significant (pvalue $=0.232)-$ the other 2 pairwise comparisons are significant (p-value $\leq$ 0.0240). The two GSMs (iECDH1ME8569_1439, and iEcDH1_1363) for E. coli ATCC DH1 shows significant difference in normalized predicted growth rate ( $\mathrm{p}$-value $=0.00112$ ). Between the 8 GSMs (iJR904, iAF1260, e_coli_core, iAF1260b, iJ01366, iML1515, iY75_1357, and iEC1372_W3110) for E. coli K-12; only
3 comparisons, (a) iAF1260 and e_coli_core (p-value $=0.287$ ), (b) e_coli_core and iAF1260b (p-value $=0.293$ ), and (c) ij01366 and iY75_1357 (p-value $=0.158$ ); are not significant - the rest of the 25 pairwise comparisons are significant ( $p$-value $\leq 0.0421$ ). The normalized predicted growth rates of the 3 GSMs (iWFL_1372, iECW_1372, and iEC1364_W) for E. coli W are not significant (p-value $\geq 0.101$ ). Finally, the normalized predicted growth rates of the 4 GSMs (iECH74115_1262, iZ_1308, iECs_1301, and iECSP_1301) for E. coli $0157: \mathrm{H} 7$ are significant (p-value $\leq 0.00112$ ).

These findings are consistent with our result comparing across all 58 GSMs (Figure 2 to 4). More importantly, these findings also show that GSMs for the same bacterial strain may not yield similar results. This may be a result of differences in experimental setup to obtain the required metabolomic data for model construction despite using the same strain as Shiratsubaki., et al. [70] had demon- 
strated that GSMs built for specific developmental stages can result in result in different prediction results. Furthermore, differences between in vivo experimental outcomes and in silico predictions from GSMs are commonplace [18,19,69,70], which had resulted in methods aiming at reconciliating such differences [71]. However such differences in GSMs may also provide a fertile basis for examining the differences between various $E$. coli strains [70] or to construct a strain-independent E. coli model [69,72].

\begin{tabular}{|c|c|c|c|}
\hline \multirow{2}{*}{ E. coli Strain } & \multicolumn{2}{|c|}{ Comparison of Growth Rates Across Various Glucose Uptake Rates } & \multirow{2}{*}{$\begin{array}{l}\text { Paired t-test } \\
\text { p-value }\end{array}$} \\
\hline & GSM 1 & GSM 2 & \\
\hline ATCC 8739 & iEcolC_1368 & iEC1349_Crooks & 0.350 \\
\hline \multirow[t]{3}{*}{ BL21 (DE3) } & iB21_1397 & iECD_1391 & 0.001 \\
\hline & iB21_1397 & iEC1356_Bl21DE3 & 0.024 \\
\hline & iECD_1391 & iEC1356_Bl21DE3 & 0.232 \\
\hline DH1 & iECDH1ME8569_1439 & iEcDH1_1363 & 0.001 \\
\hline \multirow[t]{28}{*}{$\mathrm{K}-12$} & iJR904 & iAF1260 & 0.003 \\
\hline & & e_coli_core & 0.001 \\
\hline & & iAF1260b & 0.003 \\
\hline & & iJ01366 & 0.001 \\
\hline & & iML1515 & 0.001 \\
\hline & & iY75_1357 & 0.001 \\
\hline & & iEC1372_W3110 & 0.001 \\
\hline & iAF1260 & e_coli_core & 0.287 \\
\hline & & iAF1260b & 0.022 \\
\hline & & ij01366 & $1.77 \mathrm{E}-05$ \\
\hline & & iML1515 & $1.17 \mathrm{E}-04$ \\
\hline & & iY75_1357 & $1.71 \mathrm{E}-05$ \\
\hline & & iEC1372_W3110 & $1.78 \mathrm{E}-05$ \\
\hline & e_coli_core & iAF1260b & 0.293 \\
\hline & & ij01366 & 0.001 \\
\hline & & iML1515 & 0.001 \\
\hline & & iY75_1357 & 0.001 \\
\hline & & iEC1372_W3110 & 0.001 \\
\hline & iAF1260b & iJ01366 & $1.72 \mathrm{E}-05$ \\
\hline & & iML1515 & $1.12 \mathrm{E}-04$ \\
\hline & & iY75_1357 & $1.65 \mathrm{E}-05$ \\
\hline & & iEC1372_W3110 & $1.72 \mathrm{E}-05$ \\
\hline & iJ01366 & iML1515 & 0.001 \\
\hline & & iY75_1357 & 0.158 \\
\hline & & iEC1372_W3110 & 0.036 \\
\hline & iML1515 & iY75_1357 & 0.001 \\
\hline & & iEC1372_W3110 & 0.001 \\
\hline & iY75_1357 & iEC1372_W3110 & 0.042 \\
\hline \multirow[t]{3}{*}{ W } & iWFL_1372 & iECW_1372 & 0.302 \\
\hline & & iEC1364_W & 0.286 \\
\hline & iECW_1372 & iEC1364_W & 0.102 \\
\hline \multirow[t]{6}{*}{ 0157:H7 } & iECH74115_1262 & iZ_1308 & 0.001 \\
\hline & & iECs_1301 & 0.001 \\
\hline & & iECSP_1301 & 0.001 \\
\hline & iZ_1308 & iECs_1301 & 0.001 \\
\hline & & iECSP_1301 & 0.001 \\
\hline & iECs_1301 & iECSP_1301 & 0.001 \\
\hline
\end{tabular}

Table 3: Paired t-test Analysis of Normalized Growth Rates Within Each Strain. 


\section{Conclusion}

In this study, 58 GSMs for E. coli were compared for their media components and predicted growth rates across various glucose uptake rates. Substantial differences in media components were found and our results suggest significant differences in predicted growth rates between the GSMs and even within GSMs constructed for the same strain.

\section{Supplementary Materials}

Data files for this study can be downloaded from https://bit.ly/ CompareECO_GSM.

\section{Acknowledgement}

This work was conducted as part of the Temasek Polytechnic School of Applied Science Differential Research Project and Major Project under the Student Project fund (TP_PR1052).

\section{Conflict of Interest}

The authors declare no conflict of interest.

\section{Bibliography}

1. Idalia V-MN and Bernardo F. "Escherichia coli as a Model Organism and Its Application in Biotechnology". In: Samie A, editor. Escherichia coli - Recent Advances on Physiology, Pathogenesis and Biotechnological Applications. InTech (2017).

2. Yang D., et al. "Metabolic Engineering of Escherichia coli for Natural Product Biosynthesis". Trends in Biotechnology 38.7 (2020): 745-765.

3. García-Granados R., et al. "Metabolic Engineering and Synthetic Biology: Synergies, Future, and Challenges". Frontiers in Bioengineering and Biotechnology 7 (2019): 36.

4. Murthy MV., et al. "UniKin1: A Universal, Non-Species-Specific Whole Cell Kinetic Model". Acta Scientific Microbiology 3.10 (2020): 04-08.

5. Cho JL and Ling MH. "Adaptation of Whole Cell Kinetic Model Template, UniKin1, to Escherichia coli Whole Cell Kinetic Model, ecoJC20". EC Microbiology 17.2 (2021): 254-260.

6. Comba S., et al. "Emerging Engineering Principles for Yield Improvement in Microbial Cell Design". Computational and Structural Biotechnology Journal 3.4 (2012): e201210016.

7. Cardoso JGR., et al. "Cameo: A Python Library for Computer Aided Metabolic Engineering and Optimization of Cell Factories". ACS Synthetic Biology 7.4 (2018): 1163-1166.

8. Ling MH. "AdvanceSyn Toolkit: An Open Source Suite for Model Development and Analysis in Biological Engineering". MOJ Proteomics Bioinformation 9.4 (2020): 83-86.

9. O'Brien EJ., et al. "Using Genome-scale Models to Predict Biological Capabilities”. Cell 161.5 (2015): 971-987.
10. Srinivasan S., et al. "Constructing Kinetic Models of Metabolism at Genome-Scales: A Review". Biotechnology Journal 10.9 (2015): 1345-59.

11. Simeonidis E and Price ND. "Genome-Scale Modeling for Metabolic Engineering". Journal of Industrial Microbiology and Biotechnology 42.3 (2015): 327-338.

12. Gu C., et al. "Current Status and Applications of Genome-Scale Metabolic Models". Genome Biology 20.1 (2019): 121.

13. Kim B., et al. "Applications of Genome-Scale Metabolic Network Model in Metabolic Engineering". Journal of Industrial Microbiology and Biotechnology 42.3 (2015): 339-348.

14. Kocabaş P., et al. "Analyses of Extracellular Protein Production in Bacillus subtilis - II: Responses of Reaction Network to Oxygen Transfer at Transcriptional Level". Biochemical Engineering Journal 127 (2017): 242-261.

15. Kavvas ES., et al. "Updated and Standardized Genome-Scale Reconstruction of Mycobacterium tuberculosis H37Rv, iEK1011, Simulates Flux States Indicative of Physiological Conditions". BMC Systems Biology 12.1 (2018): 25.

16. Edwards JS and Palsson BO. "The Escherichia coli MG1655 In Silico Metabolic Genotype: Its Definition, Characteristics, and Capabilities". Proceedings of the National Academy of Sciences of the United States of America 97.10 (2000): 5528-5533.

17. Ye C., et al. "Improving Lysine Production Through Construction of an Escherichia coli Enzyme-Constrained Model". Biotechnology and Bioengineering 117.11 (2020): 3533-3544.

18. Cheong KC., et al. "A Simulation Study on the Effects of Media Composition on the Growth Rate of Escherichia coli MG1655 Using iAF1260 Model". Acta Scientific Microbiology 3.8 (2020): 40-44.

19. Chang ED and Ling MH. "Explaining Monod in terms of Escherichia coli metabolism". Acta Scientific Microbiology 2.9 (2019): 66-71.

20. Mienda BS. "Escherichia coli Genome-Scale Metabolic Gene Knockout of Lactate Dehydrogenase (ldhA), Increases Succinate Production from Glycerol". Journal of Biomolecular Structure and Dynamics 36.14 (2018): 3680-3686.

21. Immanuel SRC., et al. "Integrated Constraints Based Analysis of An Engineered Violacein Pathway in Escherichia coli”. Biosystems 171 (2018): 10-19.

22. King ZA., et al. "BiGG Models: A Platform for Integrating, Standardizing and Sharing Genome-Scale Models". Nucleic Acids Research 44.D1 (2016): D515-522.

23. Feist AM and Palsson BO. "The Biomass Objective Function". Current Opinion in Microbiology 13.3 (2010): 344-349.

24. Orth JD., et al. "What is Flux Balance Analysis?" Nature Biotechnology 28.3 (2010): 245-248. 
25. Peterson JR., et al. "Parametric Studies of Metabolic Cooperativity in Escherichia coli Colonies: Strain and Geometric Confinement Effects". PLoS ONE 12.8 (2017): e0182570.

26. Orth JD., et al. "A Comprehensive Genome-Scale Reconstruction of Escherichia coli Metabolism - 2011". Molecular Systems Biology 7.1 (2011): 535.

27. Monk JM., et al. "Genome-Scale Metabolic Reconstructions of Multiple Escherichia coli Strains Highlight Strain-Specific Adaptations to Nutritional Environments". Proceedings of the National Academy of Sciences of the United States of America 110.50 (2013): 20338-20343.

28. Wang C., et al. "An Aldolase-Catalyzed New Metabolic Pathway for the Assimilation of Formaldehyde and Methanol To Synthesize 2-Keto-4-hydroxybutyrate and 1,3-Propanediol in Escherichia coli". ACS Synthetic Biology 8.11 (2019): 24832493.

29. Reed JL., et al. "An Expanded Genome-Scale Model of Escherichia coli K-12 (iJR904 GSM/GPR)". Genome Biology 4.9 (2003): R54.

30. Costa RS and Vinga S. "Assessing Escherichia coli Metabolism Models and Simulation Approaches in Phenotype Predictions: Validation Against Experimental Data". Biotechnology Progress 34.6 (2018): 1344-1354.

31. Liu L., et al. "DEF: An Automated Dead-End Filling Approach Based On Quasi-Endosymbiosis". Bioinformatics 33.3 (2017): 405-413.

32. Feist AM., et al. "A Genome-Scale Metabolic Reconstruction for Escherichia coli K-12 MG1655 that Accounts for 1260 ORFs and Thermodynamic Information". Molecular Systems Biology 3 (2007): 121.

33. Tamura T. "Grid-Based Computational Methods for the Design of Constraint-Based Parsimonious Chemical Reaction Networks to Simulate Metabolite Production: GridProd". BMC Bioinformatics 19.1 (2018): 325.

34. Orth J., et al. "Reconstruction and Use of Microbial Metabolic Networks: the Core Escherichia coli Metabolic Model as an Educational Guide". EcoSal Plus 4.1 (2010): ecosalplus.10.2.1.

35. de Arroyo Garcia L and Jones PR. "In Silico Co-Factor Balance Estimation Using Constraint-Based Modelling Informs Metabolic Engineering in Escherichia coli". PLOS Computational Biology 16.8 (2020): e1008125.

36. Apaydin M., et al. "Robust Mutant Strain Design by Pessimistic Optimization”. BMC Genomics 18.S6 (2017): 677.

37. Feist AM., et al. "Model-Driven Evaluation of the Production Potential for Growth-Coupled Products of Escherichia coli". Metabolic Engineering 12.3 (2010): 173-186.

38. Jenior ML., et al. "Transcriptome-Guided Parsimonious Flux Analysis Improves Predictions with Metabolic Networks in Complex Environments". PLOS Computational Biology 16.4 (2020): e1007099.
39. Bekiaris PS and Klamt S. "Automatic Construction of Metabolic Models with Enzyme Constraints". BMC Bioinformatics 21.1 (2020): 19 .

40. Kim D., et al. "Development of a Genome-Scale Metabolic Model and Phenome Analysis of the Probiotic Escherichia coli Strain Nissle 1917". International Journal of Molecular Sciences 22.4 (2021): 2122.

41. Chu HY., et al. "Assessing the Benefits of Horizontal Gene Transfer by Laboratory Evolution and Genome Sequencing". BMC Evolutionary Biology 18.1 (2018): 54.

42. Hosseini S-R and Wagner A. "Genomic Organization Underlying Deletional Robustness in Bacterial Metabolic Systems". Proceedings of the National Academy of Sciences of the United States of America 115.27 (2018): 7075-7080.

43. Kim H., et al. "Metabolic Network Reconstruction and Phenome Analysis of the Industrial Microbe, Escherichia coli BL21 (DE3)". PLOS ONE 13.9 (2018): e0204375.

44. Zabeti H., et al. "A Duality-Based Method for Identifying Elemental Balance Violations in Metabolic Network Models". In: $18^{\text {th }}$ International Workshop on Algorithms in Bioinformatics (WABI 2018). Schloss Dagstuhl - Leibniz-Zentrum fuer Informatik GmbH, Wadern/Saarbruecken, Germany (2018): 1-13. (Leibniz International Proceedings in Informatics (LIPIcs)).

45. Wu G. "Revelation of Yin-Yang Balance in Microbial Cell Factories by Data Mining, Flux Modeling, and Metabolic Engineering [Doctor of Philosophy]". [School of Engineering and Applied Science]: Washington University (2016).

46. Patané A., et al. "Multi-Objective Optimization of GenomeScale Metabolic Models: The Case of Ethanol Production". Annals of Operations Research 276.1-2 (2019): 211-227.

47. Gerstl MP., et al. "Exact Quantification of Cellular Robustness in Genome-Scale Metabolic Networks". Bioinformatics 32.5 (2016): 730-737.

48. Monk JM., et al. "Multi-Omics Quantification of Species Variation of Escherichia coli Links Molecular Features with Strain Phenotypes". Cell System 3.3 (2016): 238-251.e12.

49. Monk JM., et al. "iML1515, A Knowledgebase that Computes Escherichia coli Traits". Nature Biotechnology 35.10 (2017): 904-908.

50. Amin SA., et al. "Towards Creating An Extended Metabolic Model (EMM) for E. coli Using Enzyme Promiscuity Prediction and Metabolomics Data". Microbe Cell Factories 18.1 (2019): 109.

51. Hädicke 0 and Klamt S. "EColiCore2: A Reference Network Model of the Central Metabolism of Escherichia coli and Relationships to its Genome-Scale Parent Model". Scientific Report 7.1 (2017): 39647.

52. Clement TJ., et al. "Unlocking Elementary Conversion Modes: ecmtool Unveils All Capabilities of Metabolic Networks". Patterns 2.1 (2021): 100177. 
53. Heinonen M., et al. "Bayesian Metabolic Flux Analysis Reveals Intracellular Flux Couplings”. Bioinformatics 35.14 (2019): i548-557.

54. Venayak N., et al. "MoVE Identifies Metabolic Valves to Switch Between Phenotypic States". Nature Communication 9.1 (2018): 5332.

55. Serres MH., et al. "A Functional Update of the Escherichia coli K-12 Genome". Genome Biology 2.9 (2001): RESEARCH0035.

56. Gyawali R., et al. "Antimicrobial Activity of Copper Alone and in Combination with Lactic Acid against Escherichia coli 0157:H7 in Laboratory Medium and on the Surface of Lettuce and Tomatoes". Journal of Pathogens 2011 (2011): 650968.

57. Ranquet C., et al. "Cobalt Stress in Escherichia coli". Journal of Biological Chemistry 282.42 (2007): 30442-30451.

58. Majtan T., et al. "Effect of Cobalt on Escherichia coli Metabolism and Metalloporphyrin Formation". BioMetals. An International Journal on the Role of Metal Ions in Biology, Biochemistry and Medicine 24.2 (2011): 335-347.

59. Ratledge C., et al. "Effect of Iron and Zinc on Growth Patterns of Escherichia coli in Iron-Deficient Medium". Journal of Bacteriology 87 (1964): 823-827.

60. Appenzeller BMR., et al. "Advantage Provided by Iron for Escherichia coli Growth and Cultivability in Drinking Water". Applied and Environmental Microbiology 71.9 (2005): 56215623.

61. McEwan AG. "New Insights into the Protective Effect of Manganese Against Oxidative Stress". Molecular Microbiology 72.4 (2009): 812-814.

62. Baez A and Shiloach J. "Escherichia coli Avoids High Dissolved Oxygen Stress by Activation of SoxRS and Manganese-Superoxide Dismutase". Microbe Cell Factories 12.1 (2013): 23.

63. Mardare CC., et al. "Growth Inhibition of Escherichia coli by Zinc Molybdate with Different Crystalline Structures: Growth Inhibition of Escherichia coli by Zinc Molybdate". Physica Status Solidi 213.6 (2016): 1471-1478.

64. Gates AJ., et al. "Properties of the Periplasmic Nitrate Reductases from Paracoccus pantotrophus and Escherichia coli after Growth in Tungsten-Supplemented Media". FEMS Microbiology Letter 220.2 (2003): 261-269.

65. Durfee T., et al. "The Complete Genome Sequence of Escherichia coli DH10B: Insights into the Biology of a Laboratory Workhorse". Journal of Bacteriology 190.7 (2008): 2597-2606.

66. Skovgaard 0., et al. "Genome-Wide Detection of Chromosomal Rearrangements, Indels, and Mutations in Circular Chromosomes by Short Read Sequencing". Genome Research 21.8 (2011): 1388-1393.
67. Anfora AT., et al. "Uropathogenic Escherichia coli CFT073 is Adapted to Acetatogenic Growth But Does Not Require Acetate During Murine Urinary Tract Infection". Infection and Immunity 76.12 (2008): 5760-5767.

68. Fürnkranz J, ., et al. "Manhattan Distance". In: Sammut C, Webb GI, editors. Encyclopedia of Machine Learning. Boston, MA: Springer US (2011): 639-639.

69. Nouri H., et al. "A Reconciliation of Genome-Scale Metabolic Network Model of Zymomonas mobilis ZM4". Scientific Report 10.1 (2020): 7782.

70. Shiratsubaki IS., et al. "Genome-Scale Metabolic Models Highlight Stage-Specific Differences in Essential Metabolic Pathways in Trypanosoma cruzi". PLOS Neglected Tropical Diseases 14.10 (2020): e0008728.

71. van Duuren JBJH., et al. "Reconciling In Vivo and In Silico Key Biological Parameters of Pseudomonas putida KT2440 During Growth on Glucose Under Carbon-Limited Condition". BMC Biotechnology 13 (2013): 93.

72. Contador CA., et al. "Use of Genome-Scale Models to Get New Insights into the Marine Actinomycete Genus Salinispora". BMC Systems Biology 13.1 (2019): 11.

\section{Assets from publication with us}

- Prompt Acknowledgement after receiving the article

- Thorough Double blinded peer review

- Rapid Publication

- Issue of Publication Certificate

- High visibility of your Published work

Website: www.actascientific.com/

Submit Article: www.actascientific.com/submission.php Email us: editor@actascientific.com Contact us: +919182824667 